\title{
Malaysian car plate localization using region-based convolutional neural network
}

\author{
Tay Eng Liang1, Usman Ullah Sheikh², Mohd Norzali Haji Mohd ${ }^{3}$ \\ ${ }^{1}$ Intel Microelectronics (M) Sdn. Bhd., Malaysia \\ ${ }^{2}$ School of Electrical Engineering, Faculty of Engineering, Universiti Teknologi Malaysia, Malaysia \\ ${ }^{3}$ Faculty of Electrical and Electronics Engineering, Universiti Tun Hussein Onn Malaysia, Malaysia
}

\begin{tabular}{l}
\hline Article Info \\
\hline Article history: \\
Received Sep 3, 2019 \\
Revised Nov 11, 2019 \\
Accepted Dec 18, 2019 \\
\hline
\end{tabular}

Keywords:

Car plate localization

Deep learning

$\mathrm{R}-\mathrm{CNN}$

Transfer learning

\begin{abstract}
Automatic car plate localization and recognition system is a system that identifies the car plate location and recognizes the characters on the car plate input images. Within the automated system, the car plate localization stage is the first stage and is the most crucial stage as the success rate of the whole system depends heavily on it. In this paper, a Malaysian car plate localization system using Region-based Convolutional Neural Network (R-CNN) is proposed. Using transfer learning on the AlexNet CNN, the localization was greatly improved achieving best precision and recall rate of $95.19 \%$ and $97.84 \%$ respectively. Besides, the proposed R-CNN was able to localize car plates in complex scenarios such as under occlusion.
\end{abstract}

This is an open access article under the $\underline{C C B Y-S A}$ license.

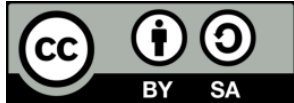

\section{Corresponding Author:}

Usman Ullah Sheikh,

School of Electrical Engineering, Faculty of Engineering,

Universiti Teknologi Malaysia, Johor Bahru, Malaysia.

Email: usman@fke.utm.my

\section{INTRODUCTION}

Automated car plate localization and recognition system is one component of an intelligent transportation system. It uses image-processing techniques to automatically identify car plates from given input images without any human intervention. This system has a very wide application area which includes traffic monitoring systems, car park access systems, traffic law enforcement systems, automatic toll payment systems, and border crossing control system [1-3]. In order to achieve a good car plate recognition result, the system must first be able to accurately localize the location of the car plate from the given input image. In general, there are two major branches of algorithms being applied to perform the automatic car plate localization task, namely algorithms based on handcrafted features and algorithms based on deep learning. Example of handcrafted features includes the location of straight lines, edge density, connectivity information, and color information. Besides, in Malaysia, there are multiple car plate formats a vailable $[4,5]$. Consequently, methods based on handcrafted features will have difficulty in handling the issue of non-standardization of Malaysian car plate formats.

In [6], the authors have proposed to localize car plate from an input image by applying Hough Transform in order to detect the location of straight lines in the image and retrieve the bounding box location based on the location of the straight lines. However, Hough Transform requires large memory and has high computation time. As for [7-9], the Sobel operator has been used to localize car plates based on vertical edge detection. The limitations of vertical edge detection are that it requires prior knowledge about the edge information in the image and it is sensitive to noise. Authors in [10] have proposed to localize car plates 
based on connected components analysis (CCA) while authors in [11] have proposed to use image binarization with the Otsu method. CCA may generate broken objects while the Otsu method is less accurate when both car body and car plate have the same color.

Deep learning approach, or also known as deep structured learning or hierarchical learning [12], replaces the need for handcrafted features [13] as the process of determining and learning features is achieved by the algorithm through a large number of training images [14]. There are various deep learning architectures available, which include Convolutional Neural Network (CNN), Deep Belief Network (DBF) and Deep Stacking Network (DSN) [12]. Each architecture has its own application area, for instance, CNN is suitable for object recognition [15]. Previous literatures have applied deep learning algorithm to localize car plates [14, 16-18] using CNN but have some limitations such as high false negative rate as low-resolution dataset has been used in the training process, a ssumption that there is only one car plate per image and they did not consider the complexity of Malaysian car plates, therefore, not suitable to be applied for Malaysian car plate localization system. Hence, a Malaysian car plate localization system using Region-based Convolutional Neural Network (R-CNN) is proposed. By using this deep learning approach, the dependency on handcrafted features can be eliminated and the issue of non-standardization of Malaysian car plate formats can be handled by the proposed system. The proposed system also aims to be able to localize all car plates from an input image if there are multiple car plates exists in the image. The rest of this paper is organized as follows. Section II describes the proposed algorithm, tools used and evaluation criteria used to evaluate the developed system. Results, analysis, and discussions are presented in Section III. Finally, the conclusion and recommendations for future work are discussed in Section IV.

\section{CAR PLATE LOCALIZATION WITH R-CNN}

The scope of this work is on Malaysian car plates with a black background and white characters for normal cars as well as a white background and black characters for taxies. The circumstances that are considered include multiple car plates (up to 3 car plates per image), special car plates (Putrajaya, Perodua, etc.), car plates with other logos, partially blocked car plates and car plates captured at an angle.

\subsection{Image acquisition and preprocessing}

The image acquisition process is carried out in order to collect the input dataset required to train and test the proposed system. In this work, a total of 1.157 Malaysian car plate images were acquired consisting of front and rear car images at 13 Megapixel resolutions in normal daylight condition. Each car plate image consists of a minimum of 1 car plate and up to a maximum of 3 car plates. The car plates in the input image take up approximately $20 \%$ to $30 \%$ of the whole image area. Images were also captured at different angles with respect to the camera with a camera angle of between $0^{\circ}$ to $30^{\circ}$. Images are first converted to grayscale as the Malaysian car-plate consists of only black and white colors. Next, images are resized to $826 \times 465$ from the original resolution $4128 \times 2322$ - to reduce compute time while maintaining car plate image quality. After that, median filter is applied to the grayscale images in order to remove image noise [19] before a pplying image intensity adjustment to enhance the contrast.

\subsection{Training the AlexNet}

AlexNet is a pre-trained CNN network that has been created by Alex Krizhevsky et al. [20]. It is an 8 layer $\mathrm{CNN}$ model that has been trained with a total of 1.2 million training images obtained from ImageNet Large Scale Visual Recognition Challenge 2010 (ILSVRC-2010) with 1.000 object classes. $\mathrm{R}-\mathrm{CNN}$ is a network that combines CNN network with region proposals [21], which has been proven to be successful for semantic segmentation [22] and object detection [23]. In order to perform transfer learning based on AlexNet, the last few layers of the AlexNet are replaced with the layers that are applicable for the current task. The last fully connected layer is changed to achieve two output classes, which are; car plate class and the background class. Transfer learning is then carried out in 3 iterations. In the first iteration, ground-truths are labeled by enclosing only the car plate numbers. The purpose of doing so is to allow the R-CNN network to learn the car plate characters. Figure 1(a) shows an example of labeled ground-truth for transfer learning in iteration 1. In this iteration, a total of 693 labeled training images (training set 1) have been used to train the R-CNN network. In the second iteration, transfer learning is done based on the trained network obtained from iteration 1 (itr1_net). Firstly, itr1_net has been used to perform car plate localization on another set of training dataset (training set 2), which is made up of 232 training images. Then, based on the localization results on training set 2, all the network's false positive locations are labeled on training set 2 as background class while the true car plate locations have been labeled as car plate class. The labeled training set 2 is then used to train the R-CNN network in this second iteration of transfer learning. By doing so, it reduces the false positive detection of the network. Lastly, further training is carried out based 
on the trained network obtained from iteration 2 (itr2_net). In this iteration, 300 training images from the training set 1 are relabeled so that both the car plate edges and the car plate characters are enclosed by the ground-truth bounding boxes. The purpose of doing so is to allow the network to learn that the car plate numbers are actually located on car plates. The relabeled training images are used to perform transfer learning on the itr2 net.

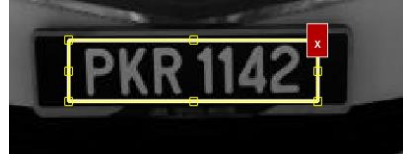

(a)

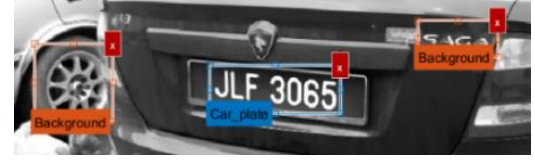

(b)

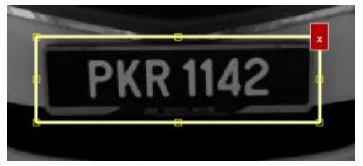

(c)

Figure 1. (a-c) Examples of labeled ground truth images used during training in iterations 1, 2, 3 respectively

\subsection{Post-processing}

The post-processing stage is required to perform filtering on the generated bounding boxes by the R-CNN network during car plate localization testing phase. Firstly, confidence filtering stage is applied to the generated bounding boxes. The R-CNN network produces bounding boxes as well as the localization confidence factor for each of the classified car-plate region. The generated bounding boxes is filtered with a preset confidence threshold $t$, where only the bounding boxes with a confidence factor greater than the threshold value will be taken as the localization results. The threshold that has been chosen is 0.92 , where it has been obtained experimentally by executing car plate localization on training set 2 . If there are cases where none of the generated bounding boxes having a confidence factor greater than 0.92 , the next highest confidence bounding box is selected. By applying this filtering stage, the false positive regions detected by the R-CNN network can be effectively eliminated. After that, all overlapping bounding boxes, where any bounding boxes with intersection over union (IoU) ratio greater than threshold $r$ are merged. The threshold $r$ that has been chosen is 0.15 . As for the localization confidence factor of the resultant merged bounding box, it is calculated as the average confidence of all the overlapping bounding boxes.

\subsection{Evaluation method}

In order to evaluate the performance of the proposed system, several evaluation criteria are applied, namely the number of true positives (TP), false positives (FP), false negatives (FN), precision rate and recall rate [14]. These criteria have been used to quantify the accuracy of the car plate localization results for the proposed system. TP is referred to as the detected regions that contain real car plates. In this paper, the IoU ratio between the detected regions and the car plate ground-truth regions have been computed during the evaluation stage. The detected regions are considered as TP if the IoU ratio between the detected regions and the ground-truth is greater than 0.4 . FP refers to the detected regions which do not contain any car plate. FP shows the number of regions that have been incorrectly detected. FN refers to the miss rate i.e. real car plates that have not been detected by the system. We define the precision rate as the totalnumber of correctly detected car plates over the total number of detected regions. It has been used to provide an insight into the number of false alarms. A system with low precision rate will generate many bounding boxes with only a few of them containing correctly detected car plates. We also define recall as the total number of correctly detected car plates over the total number of ground-truth. This criterion gives information on the amount of ground-truth car plates that have been successfully detected. If the system has been unable to localize the majority of the ground-truth car plates, it will have a low recall rate.

\subsection{Creating a benchmark}

Two different approaches have been used to train another $2 \mathrm{R}-\mathrm{CNN}$ networks for car plate localization. The car plate localization results produced by the $2 \mathrm{R}-\mathrm{CNN}$ networks are then being used as a benchmark to compare with the results produced by AlexNet trained with transfer learning. In the first approach, R-CNN network has been trained from scratch by using the captured car plate images. In this approach, a 5 layer CNN model has been built, which consists of 3 convolutional layers and 2 fully connected layers. Similar to the transfer learning approach, the last fully connected layer is made up of two neurons in order to output 2 classes, which are the car plate class and also the background class. In this approach, the R-CNN network has been trained with a total of 693 training images, which is about $60 \%$ of the totalcaptured images. In the second approach, transfer learning based on CIFAR -10 network has been 
carried out. CIFAR-10 network is a pre-trained network that has been created by Alex Krizhevsky [24]. It is a 5 layer $\mathrm{CNN}$ model that has been trained with 50.000 of training images. The training images are made up of 10 classes, which are cat, dog, bird, horse, frog, deer, airplane, ship, automobile and truck. In this transfer learning approach, the last few layers of the pre-trained network have been replaced with the layers that suit for car plate localization. The first fully connected layer has been changed to 256 neurons instead of the original 64 neurons in order to allow the network to relate more learned features to the characteristics in different classes. As for the last fully connected layer, it has been changed to 2 neurons instead of 10 neurons, which translates to 2 output classes, car plate, and background. Similarly, in this approach, the R-CNN network has also been trained with 693 training images.

\section{RESULTS}

There are a total of 3 training approaches being applied in this work, namely, training a new network from scratch (approach 1), transfer learning based on CIFAR-10 network (approach 2), and tra nsfer learning based on AlexNet (approach 3). Figure 2 shows the training graphs for all three approaches. Mini-batch is the number of training images to be propagated through the network during each iteration. One mini-batch is a subset of the total training images a vailable. When all the mini-batches have completely passed through the network, it is referred to as one epoch. For the new network that is trained from scratch, the total training time is 4.2 hours. From the training graph in Figure 2(a), it has been observed that the mini-batch loss of the network saturates at 0.45 while the mini-batch accuracy saturates at $80 \%$. This is because the amount of training images available in this project is considerably small and hence, the network accuracy cannot improve further with this number of training images.

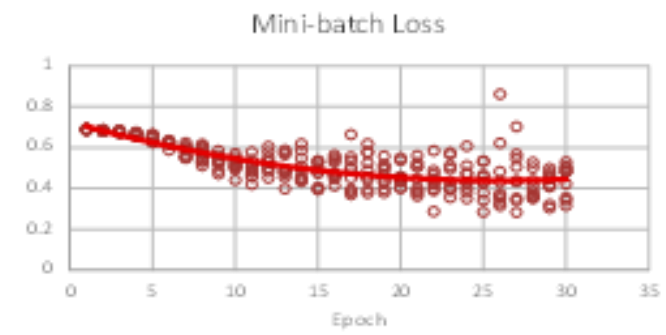

Mini-batch Loss

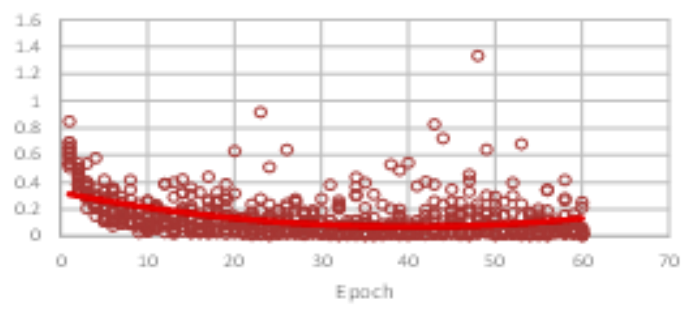

Mini-batch Loss

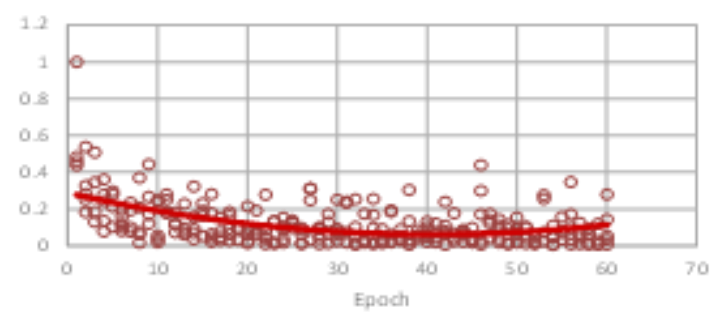

(a)
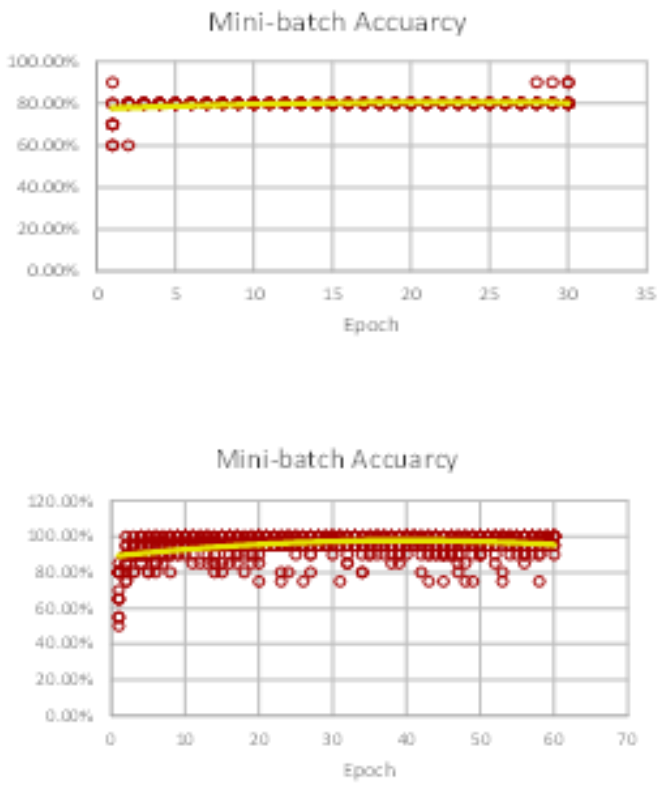

(b)

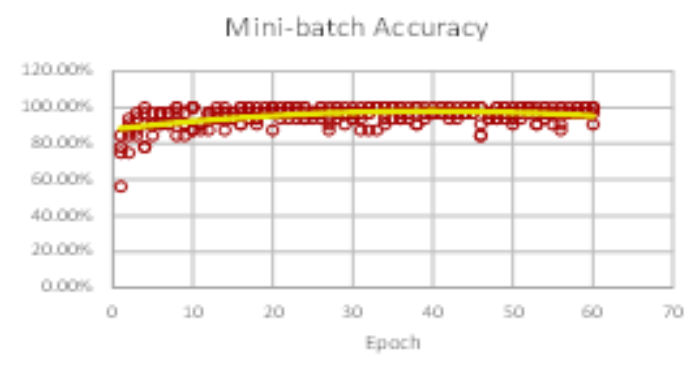

(c)

Figure 2. (a-c) Mini-batch loss and mini-batch accuracy training graphs in approaches 1,2, 3 respectively 
As for the CIFAR-10 network, the training time is 0.52 hour. The training time for this approach is shorter because the network is running on an image input layer of smaller size. As a result, fewer multiply-accumulate operations have been performed and less amount of memory space was needed. From the training graph in Figure 2(b), it can be seen that the average mini-batch loss for the network is at around 0.15 while the avera ge mini-batch accuracy is at around $95 \%$, which outperforms the first approach. Figure 2(c) shows the training graph for transfer learning based on AlextNet in iteration 3. A total of 5.9 hours was taken to train the R-CNN network in this iteration. The time taken to train this network is relatively long as the AlexNet is a big neural network that contains more layers and the image input layer size is relatively bigger. As a result, more multiply-accumulate operations are performed and a higher amount of memory space was used. Therefore, the total training time is longer. As illustrated in the training graph, the mini-batch loss approaches 0 while the mini-batch accuracy approaches $100 \%$, which reflects that the trained network is able to achieve high accuracy.

\subsection{Car plate localization results}

In this work, a total of 232 test images were used to test the developed car plate localization system (around $20 \%$ of the total acquired images in this work). Figure 3 shows the AlexNet car plate localization results for car plates captured in different situations. The proposed approach is able to localize car-plates in different situations accurately, e.g. even for incomplete car-plate (missing 1 car-plate character), special Malaysian car-plate, multiple car-plates, occlusion and at an angle, as shown in Figure 3. Table 1 shows the results of the evaluation criteria set. The average precision and recall are calculated by averaging the precision and recall results for all test images. The "total detected regions" is the sum of bounding boxes produced by the car plate localization system. The "average IoU with TP" is the average amount of intersection between the detected TP with the manual labeled ground-truths. As for the "requires merging" criteria, it is the total number of bounding boxes that require d merging to be performed. Lastly, the "avera ge. TP confidence" is calculated by averaging the confidence factors for each TP bounding boxes.

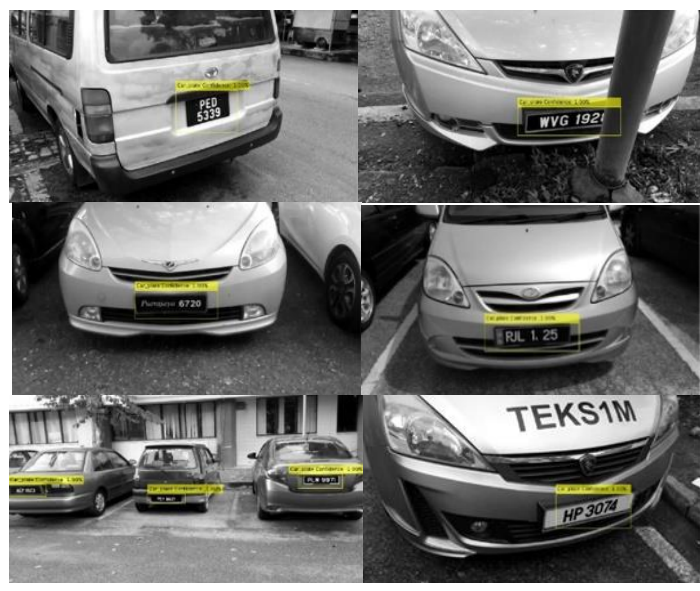

Figure 3. Carplate localization results

Table 1. Evaluation criteria results

\begin{tabular}{|c|c|c|c|c|}
\hline & \multirow{2}{*}{$\begin{array}{l}\text { Proposed } \\
\text { network }\end{array}$} & \multirow{2}{*}{$\begin{array}{c}\text { CIFAR } \\
10\end{array}$} & \multicolumn{2}{|c|}{ AlexNet } \\
\hline & & & Iter. 1 & Iter. 3 \\
\hline Average Precision (\%) & 19.4 & 82.22 & 93.64 & 95.19 \\
\hline Average Recall (\%) & 18.32 & 86.28 & 97.92 & 97.84 \\
\hline Total detected regions & 232 & 295 & 290 & 289 \\
\hline $\begin{array}{l}\text { Total true positives } \\
\text { (TP) }\end{array}$ & 45 & 222 & 258 & 265 \\
\hline $\begin{array}{l}\text { Total false positives } \\
\text { (FP) }\end{array}$ & 187 & 73 & 32 & 24 \\
\hline $\begin{array}{l}\text { Total false negatives } \\
\text { (FN) }\end{array}$ & 226 & 54 & 14 & 11 \\
\hline Average IoU with TP & 0.6061 & 0.6588 & 0.7525 & 0.7346 \\
\hline Requires merging & 0 & 83 & 32 & 5 \\
\hline $\begin{array}{l}\text { Average TP } \\
\text { confidence }\end{array}$ & 0.5648 & 0.9856 & 0.9950 & 0.9964 \\
\hline
\end{tabular}

Based on the results, it shows that the average precision and recall rate had increased from training new network from scratch (approach 1) to three-iteration transfer learning based on AlexNet (approach 3). The same trend has been observed in the totalnumber of TP. This indicates that the system performance had improved when moving from approach 1 to approach 3. On the other hand, the amount of FP and FN had decreased from approach 1 to a pproach 3 . This has shown the system has been able to produce bette r qua lity results with lesser noise. Besides, the average IoU between TP and ground-truths have been recorded to be at around 0.6 to 0.75 , with the highest value achieved in approach 3 . This has shown that the developed system in approach 3 has been able to produce bounding boxes that closely matched the labeled ground-truth. From the evaluation criteria results, it can be observed that the highest precision and recall rates have been achieved in approach 3 , which are $95.19 \%$ and $97.84 \%$.

The same trend is observed in the totalnumber of TP. Out of the 289 detected regions in approach 3 , there are 265 regions are true positives, which is $91.7 \%$ out of the total detected regions. This shows that the proposed method is able to localize car-plates with low false detections. The average IoU between TP and ground-truths have been recorded to be at 0.7346 . In addition, it has been observed that the totalnumber of bounding boxes that required merging in approach 3 is very low. This means that the R-CNN network 
is able to produce bounding boxes that completely encloses the car plate regions and less likely to require the merging stage. As for the average TP confidence, the trained network is been able to achieve very high confidence values for the detected TP, close to 1 .

\subsection{Analysis}

Based on the evaluation results, it can be seen that the R-CNN network has produced accurate car plate localization results. Further training the AlexNet in iteration 3 was able to produce even better results as compared to the first iteration. This is because the network in the third iteration has now learned that car-plate numbers are found on car plates instead of just learning to recognize characters and numbers. As a result, this has assisted the car plate localization system to identify the region of car plates within the input images. The distribution graph of TP confidence factors for AlexNet in iteration 3 is shown in Figure 4 further proves the localization accuracy of the network. From the distribution results, it can be seen that most of the confidence factors for the TP are greater than 0.99. It reflects that the iteration 3 network is able to produce accurate localization results with high confidence factor on most of the test images. When comparing the AlextNet result to CIFAR-10 network result, it can be observed that the CIFAR-10 network has relatively lower precision and recall rate. This is because AlexNet is a huge neural network that has deep layers and has been trained with a very large amount of training dataset (1.2 million images) in the previous training. As a result, AlexNet has a higher number of features being learned from the previous training a compared to CIFAR-10 network, which is being trained with 50.000 images.

On the other hand, for the newly built network that has been trained from scratch, weaker results have been produced, where it has lower precision and recall rate. This is because the amount of training data set a vailable for training the R-CNN network is considerably small as compared to the a mount of training dataset that the pre-trained networks have been trained on. As a result, without performing transfer learning, purely training the new network from scratch with the small amount of dataset has resulted in lower car pla te localization accuracy. This shows that if the amount of training dataset is not sufficient, it is preferable to perform transfer learning instead of training a new network from scratch. Even though the AlexNet in iteration 3 has been able to produce accurate car plate localization results, there are still some false positives that have been detected as shown in Figure 5. Generally, the false positives detected by the system can be divided into two main types, which are the detection of car model names and the background regions. Some of the car model names are incorrectly detected as the car plate region as the car model name regions also contained characters and numbers, which are very similar to the car plate regions. On the other ha nd, background regions such as car grilles have also been incorrectly detected as car plate regions. This is because the regions of the car grilles contained a well-defined boundary, which is identical to carplates.

\section{TP confidence factor distribution for AlexNet (iteration 3)}

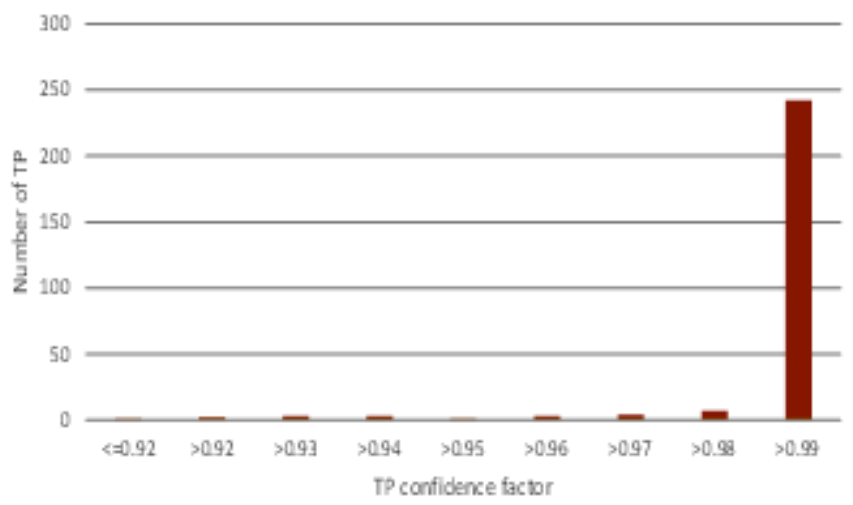

Figure 4. Distribution graph of TP confidence factor for iteration 3 network

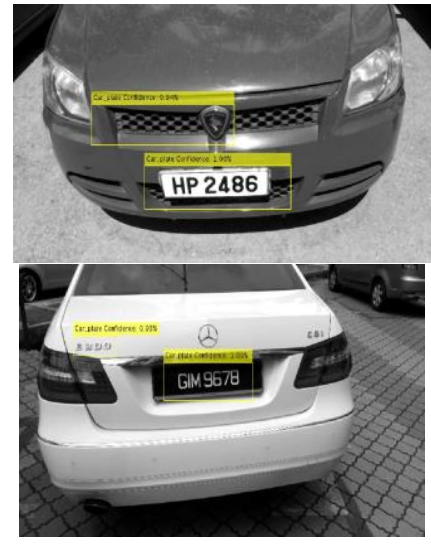

Figure 5. Examples for false positives

\subsection{Comparison with existing work}

In order to benchmark the developed car plate localization system, comparison with previous work has been performed. Currently, there is no deep learning based work that has been done on Malaysian car-plates. Besides, none of the previous work is using R-CNN approach to perform car plate localization. 
Therefore, a comparison has been done against the previous work that is not exactly the same but closest to the current work. Even though there are some limitations on this comparison as it is not one to one comparison, but it served as an indicator to provide a general view on the current standing of this work. The previous work done by Hui Li and Chunhua Shen [14] has been chosen as the benchmark work for comparison. Table 2 illustrates the comparison between [14] with the current work. The authors in the previous work have carried out an evaluation on their system by running a car plate localization process on Caltech dataset [25].

The Caltech dataset is made up of 126 US car plate images. All the images in the dataset are the images of cars captured from the rear and acquired in the parking lots of California Institute of Technology (Caltech). In order to compare the current work with the previous work, the proposed work was also tested on the Caltech dataset. Based on the precision and recall rates as shown in Table 2, even though the proposed method was purely trained based on Malaysian car plates, the system is still able to produce comparable results to the previous work when applied on the Caltech dataset. This shows that the R-CNN in the current work is sufficiently generalized to be able to work on different datasets. As for execution performance, the processing time for the previous work in [9] (NVIDIA Tesla K40c, 6GB) is $5 \mathrm{sec}$, while the proposed work's processing times are $18 \mathrm{sec}$ (NVIDIA GeForce 940MX, 4GB) and $4.5 \mathrm{sec}$ (NVIDIA GeForce GTX1060, 6GB) respectively. Re-running the proposed work on NVIDIA GeForce GTX1060 is able to achieve similar processing time as compared to previous work. This has shown that the deep learning algorithm processing time is heavily dependent on the hardware resources available. Figure 6 shows some examples of localization results for the proposed method on the Caltech dataset. It can be observed that the proposed method has been able to accurately localize car-plates from the input images even though the images are having a US plate format.

Table 2. Comparison with previous work

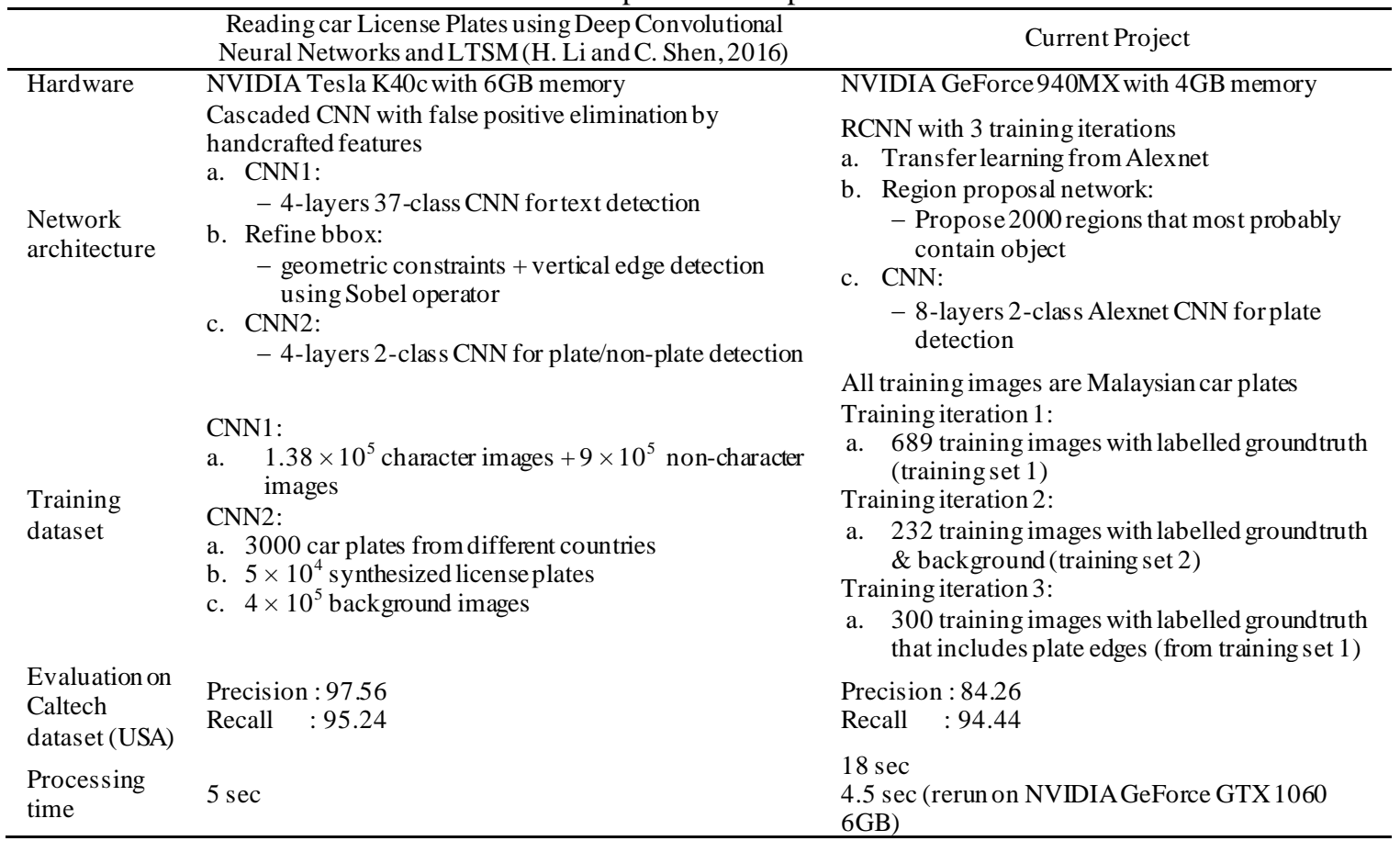
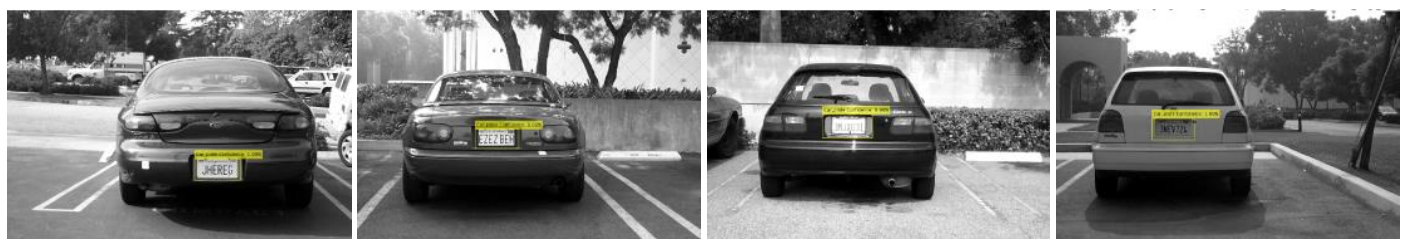

Figure 6. Carplate localization results on the Caltech data set 


\section{CONCLUSION}

In this work, a Malaysian car plate localization system has been successfully developed using deep learning approach. The developed car plate localization system has been able to accurately localize the car plate location from input images by creating bounding boxes around the car plates. The images used in this work include images with multiple car plates, special character car plates, car plates with logos on it, partially blocked car plates and car plates captured at different angles. There are several potential improvements and modifications that can be done to further enhance the current car plate localization system. Firstly, a car plate number recognition stage can be added to the end of the car plate localization stage. By adding the car plate number recognition stage, the car plate numbers in the localized regions can be recognized and at the same time, any "background" type of false positives produced by the localization stage can be eliminated effectively. In addition, a large number of car model name images can be collected and be used to re-train the R-CNN network as a background class. This can effectively help the network to reject those text-based false positive regions especially the regions containing car model name. By doing so, the syste $\mathrm{m}$ can be made more robust as it will now be able to work in different weather and lighting conditions.

\section{ACKNOWLEDGEMENTS}

The authors thank the Ministry of Education Malaysia and Universiti Tun Hussein Onn (UTHM) for their support under the Fundamental Research Grant Scheme (FRGS), grant number 1580 and Universiti Teknologi Malaysia under Research University Grant (GUP), grant number 19H61.

\section{REFERENCES}

[1] P. I. Reji and V. S. Dharun, "License Plate Localization: A Review," International Journal of Engineering Trends and Technology (IJETT), vol. 10, no. 13, pp. 604-615, Apr. 2014.

[2] T. K. Lai, A. F. Abbas, A. M. Abdu, U. U. Sheikh, M. Mokji, and K. Khalil, "Super Resolution of Car Plate Images U sing Generative Adversarial Networks," 2019 IEEE 15th International Colloquium on Signal Processing \& Its Applications (CSPA), Penang, Malaysia, pp. 80-85, 2019.

[3] J. Tai, S. Tseng, C. Lin, and K. Song, "Real-Time Image Tracking for Automatic Traffic Monitoring and Enforcement Applications," Image and Vision Computing, vol. 22, no. 6, pp. 485-501, June 2004.

[4] S. Setumin, U. U. Sheikh, and S. A. R. Abu-Bakar, "Character-Based Car Plate Detection and Localization," 10th International Conference on Information Science, Signal Processing and their Applications (ISSPA 2010), Kuala Lumpur, pp. 737-740, 2010.

[5] S. Setumin, U. U. Sheikh, and S. Member, "Car Plate Character Extraction and Recognition using Stroke Analysis Computer Vision, Video and Image Processing Lab., Dept.. of Microelectronics and Computer," 2010 Sixth Int. Conf. Signal-Image Technol. Internet Based Syst., pp. 30-34, 2010.

[6] V. Ganapathy and W. L. D. Lui, "A Malaysian Vehicle License Plate Localization and Recognition System," Journal Systemics, Cybernetics and Informatics, vol. 6, no. 1, pp. 13-20, 2008.

[7] P. Tarabek, "A Real-Time License Plate Localization Method Based on Vertical Edge Analysis," 2012 Federated Conference on Computer Science and Information Systems (FedCSIS), Wroclaw, pp. 149-154, 2012.

[8] S. Saha and S. Basu, "License Plate Localization from Vehicle Images: An Edge Based Multi-Stage Approach," International Journal of Recent Trends in Engineering, vol. 1, no. 1, pp. 284-288, May 2009.

[9] M. H. Dashtban, Z. Dashtan, and H. Bevrani, "A Novel Approach for Vehicle License Plate Localization and Recognition," in International Journal of Computer Applications, vol. 26, no. 11, pp. 22-30, July 2011.

[10] H. Kwaśnicka and B. Wawrzyniak, "License Plate Localization and Recognition in Camera Pictures," Artificial Intelligence Methods, pp. 243-246, November 2002.

[11] B. V. Ghaffari, T. Kitajima, and M. Kouhnavard, "A New Localization Method for Malaysian License Plate," GSTF J. Comput., vol. 2, no. 4, 2013.

[12] L. Deng and D. Yu, "Deep Learning: Methods and Applications," Foundations and Trends ${ }^{\circledR}$ in Signal Processing, vol. 7, no. 3-4, pp. 197-387, 2013.

[13] Y. LeCun, Y. Bengio, G. Hinton, L. Y., B. Y., and H. G., "Deep Learning," Nature, vol. 521, no. 7553, pp. 436-444, May 2015.

[14] H. Li and C. Shen, "Reading Car License Plates using Deep Convolutional Neural Networks and LSTMs," Arxiv, pp. 1-17, Jan. 2016.

[15] S. A. Radzi and M. Khalil-Hani, "Character Recognition of License Plate Number using Convolutional Neural Network," Visual Informatics: Sustaining Research and Innovations (IVIC), pp. 45-55, January 2011.

[16] A. Naimi, Y. Kessentini, and M. Hammami, "Multi-nation and Multi-norm License Plates Detection in Real Traffic Surveillance Environment using Deep Learning," International Conference on Neural Information Processing Neural Information Processing, vol. 9948, pp. 462-469, September 2016.

[17] M. Earl, "Number plate recognition with Tensorflow." [Online]. Available: https://matthewearl.github.io/2016/05/06/cnn-anpr/. [Accessed: 16-Nov-2016].

[18] C. Gerber and M. Chung, "Number Plate Detection with a Multi-Convolutional Neural Network Approach with Optical Character," in Journal of Information Processing Systems, vol. 12, no. 1, pp. 100-108, March 2016. 
[19] The Mathworks, "Image Processing ToolboxTM User's Guide R2016b," 2016.

[20] A. Krizhevsky, I. Sutskever, and G. E. Hinton, "ImageNet Classification with Deep Convolutional Neural Networks," in Advances in neural information processing systems, vol. 25, no. 2, pp. 1-9, Januay 2012.

[21] R. Girshick, J. Donahue, T. Darrell and J. Malik, "Region-Based Convolutional Networks for Accurate Object Detection and Segmentation," in IEEE Transactions on Pattern Analysis and Machine Intelligence, vol. 38, no. 1, pp. 142-158, 1 Jan. 2016.

[22] J. Carreira and C. Sminchisescu, "CPMC: Automatic Object Segmentation Using Constrained Parametric Min-Cuts," in IEEE Transactions on Pattern Analysis and Machine Intelligence, vol. 34, no. 7, pp. 1312-1328, July 2012.

[23] J. Uijlings, K. van de Sande, T. Gevers, and A. Smeulders, "Selective Search for Object Recognition," International Journal of Computer Vision, vol. 104, no. 2, pp. 154-171, April 2013.

[24] A. Krizhevsky, "Learning Multiple Layers of Features from Tiny Images," Sci. Dep. Univ. Toronto, pp. 1-60, April 2009.

[25] C. Computational Vision Group, "Computational Vision: Archive," $1999 . \quad$ [Online]. Available: http://www.vision.caltech.edu/html-files/archive.html. [Accessed: 28-May-2017].

\section{BIOGRAPHIES OF AUTHORS}
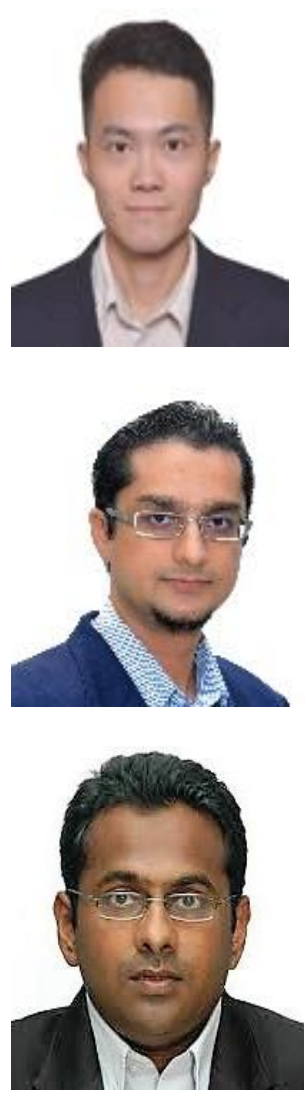

Tay Eng Liang received the Bachelor of Electrical and Electronics Engineering (Hons.) from University Tenaga Nasional, Malaysia, in 2015, and Master of Engineering in Computer and Microelectronic Systems from Universiti Teknologi Malaysia, Malaysia, in 2017. He was the recipient of Industry Excellence Award of University Tenaga Nasional in 2015 and Best Student Award from Universiti Teknologi Malaysia in 2017. In 2015, he has joined Intel Microelectronics (M) Sdn. Bhd, Penang, Malaysia, as a design engineer. He has been working on silicon design and involved in designing the next generation desktop processor. He is focusing in static timing analysis domain and is leading the timing convergence activity for the design.

Usman Ullah Sheikh received his $\mathrm{PhD}$ degree (2009) in image processing and computer vision from Universiti Teknologi Malaysia. His research work is mainly on computer vision, machine learning and embedded systems design. He is currently a Senior Lecturer at Universiti Teknologi Malaysia.

Mohd Norzali Haji Mohd is currently working as Senior Lecturer, Faculty Lab Manager (HoD) and Former Industrial Training Coordinator at the Department of Computer Engineering, Faculty of Electrical and Electronic Engineering, Universiti Tun Hussein Onn Malaysia (UTHM). He received B.Eng., M.Eng. from Fukui University, Japan in 2002 and 2004 respectively. In April 2015, he received Ph.D. from the Department of Information Sciences and Biomedical Engineering, Kagoshima University, Japan. His research interests are computer vision, robot vision, pattern recognition and image processing. 